\title{
Dónde y cómo buscar información para mi paciente: MEDLINE
}

¿Qué es MEDLINE? (MEDlars onLINE):

MEDLINE es la base de datos bibliográfica más importante en el campo de la biomedicina, producida por la Biblioteca Nacional de Medicina de los Estados Unidos (National Library of Medicine, o NLM.). Es el equivalente electrónico del "Index Medicus", así como de las citas de la lista especial de publicaciones (la cual incluye el Index to Dental Literature y el International Nursing Index). La misma contiene más de 8.400 .000 referencias de artículos médicos de más de 3.800 publicaciones periódicas de todo el mundo, por lo cual es la base de datos de referencia bibliográfica biomédica más grande y la más extensamente consultada. Posee información desde el año 1966 hasta la actualidad. La publicación se actualiza mensualmente, y cada mes se incorporan a la base aproximadamente 31.000 nuevas citas. No es una base de datos de texto completo, así que no ofrece el documento entero que se busca. Sin embargo el $76 \%$ de todas las referencias posee resúmenes estructurados (abstracts) de los artículos originales. Tanto las búsquedas en esta base de datos como las referencias encontradas se encuentran exclusivamente en idioma inglés, por lo que cierto dominio del inglés médico es requerido para su máximo aprovechamiento.

\section{Breve reseña histórica:}

MEDLINE es un producto de la NLM de Estados Unidos. Esta institución se fundó en 1836, como Biblioteca de Medicina del Ejército. Su período de crecimiento importante comenzó en 1865 bajo la dirección del Dr. John Shaw Billings. Durante los 30 años que permaneció como director, se convirtió en la mayor biblioteca médica del país, realizando entonces una importante labor referente a la organización, almacenamiento y el acceso a la literatura biomédica. Primero desarrolló una amplia colección de los boletines médicos mundiales, luego elaboró un índice (similar al de los libros) para los artículos de los bo- letines, a continuación incluyó encabezamientos de los temas en el contenido biográfico de búsqueda fácil y por último la publicación mensual de esta tabla llamada "Index Medicus". El contenido total de esta publicación conforma la Base de Datos Biomédica MEDLINE.

Después de la Segunda Guerra Mundial, la Biblioteca, conocida entonces como la Army Medical Library and Museum, empezó a llamarse Armed Forces Medical Library, siendo transferida posteriormente al Departamento de Sanidad. Desde 1960 comparte el campus de los National Institutes of Health en Bethesda (Maryland). En 1966 se abrió a la consulta on-line el antiguo repertorio bibliográfico Index Medicus con el nombre de MEDLINE, que recopila, además, las referencias del International Nursing Index (índice de recursos de enfermería), y el Index to Dental Literature (de salud dental).

En un principio las búsquedas en esta base eran realizadas manualmente. La recolección de datos y la elaboración del Index Medicus continúa hasta la actualidad y se convirtió en la tarea más intensa de la NLM. En 1958 se adoptó el procesamiento electrónico de datos para la elaboración del Index y un sistema de cintas magnéticas para realizar las búsquedas a través del sistema MEDLARS (MEDical Literature Analysis and Retrieval System, Sistema de Búsqueda y Análisis de la Literatura Médica). En 1970 varias bibliotecas médicas de los Estados Unidos y algunas de Europa y Asia brindaban el servicio de búsquedas por medio de estas cintas magnéticas. En 1989 varias empresas privadas comenzaron a producir el MEDLINE en formato de CD-ROM para las bibliotecas médicas de todo el mundo. Por último, en junio de1997, la N.L.M. puso la base para consultas gratuitas vía Internet.

El sistema de búsquedas MEDLARS es una terminología general para más de 20 bases de datos, cada una con alcances definidos, por ejemplo:

MEDLINE: Citas bibliográficas de los artículos de las publicaciones biomédicos.

CANCERLIT: Citas bibliográficas de los artículos referentes al cáncer.

AIDSLINE: Citas bibliográficas de los artículos referentes al SIDA.

AGELINE: Citas bibliográficas de los artículos referentes a geriatría.

AVLINE: Citas bibliográficas de material audiovisual sobre clínica médica.

BIOETHICS: Citas bibliográficas sobre ética y política pública en la salud y en la investigación biomédica.

HEMLINE: Diccionario de más de 650.000 sustancias químicas.

HISLINE: Historia de la medicina y las ciencias relacionadas.

HEALTH: Citas bibliográficas sobre prestaciones de salud. POPLINE: Citas bibliográficas sobre la población y al planeamiento familiar.

TOXLINE: Citas bibliográficas sobre los efectos toxicológicos, farmacológicos, bioquímicos y psicológicos de las drogas y otros químicos.

TOXNET: Sistema de bases de datos orientadas a la toxicología.

PDQ: Los últimos tratamientos del cáncer en los Estados Unidos

Para mayor información sobre todas las bases, dirigirse a MEDLARS en internet.

(http://www.nlm.nih.gov/pubs/factsheets/online databases.html). 


\section{¿Qué son los MeSH, o Medical Subjet Headings (Encabezados de Te- mas Médicos)?}

Todas las bases de datos presentan una estructura interna dividida en campos indexados y combinables entre sí que facilitan la búsqueda; MEDLINE sigue el mismo esquema y nos permite buscar en todos los campos que contiene. Algunos ejemplos de campos son el título de artículo, los autores, la publicación, la fecha, las palabras clave, etc. No todos estos campos se pueden usar en los distintos programas que ofrecen MEDLTNE (tanto en CD-ROM como vía Web); algunos de ellos no permiten la búsqueda en dichos campos, aunque siempre los mostrarán cuando aparezca la referencia bibliográfica. Pero como normalmente lo que se pretende es encontrar información sobre un tema determinado, la mejor herramienta a usar es el Tesauro ó Diccionario, una relación de todos los descriptores ó palabras-clave (keywords) que se utilizan para indexar una base de datos. En el caso de MEDLINE, el tesauro se conoce como MeSH (Medical Subject Headings).

La facilidad de acceso a la base de datos MEDLINE en soporte CD-ROM ó via Web, de cualquiera de las distintas versiones que se encuentran en el mercado, da pie a que el médico realice directamente sus consultas en lenguaje natural sin preocuparse demasiado si está utilizando los descriptores o palabras clave adecuadas para recuperar la información que realmente le interesa. Esta práctica conlleva evidentemente el riesgo de no encontrar información sobre el tema que buscamos, perderse artículos relevantes o bien tener que dedicar bastante tiempo a depurar manualmente la gran cantidad de citas recuperadas. Una herramienta muy útil, que puede ayudar a subsanar en parte estos peligros y a plantear correctamente una adecuada estrategia de búsqueda, es el Medical Subject Headings, más conocido por la abreviatura MeSH. El mismo constituye el tesauro (o diccionario de terminología controlada) de la base de datos MEDLINE. Es el conjunto de todos los descriptores o palabras clave, cerca de 18.000, que forman el vocabulario controlado y dinámico de los términos que se utiLizan tanto para la indexación de un documento como para su posterior recuperación. Cada año se incorporan términos nuevos, y otros ya existentes se modifican en función de los nuevos conceptos y alcance de la literatura médica. La misión que cumplen estos descriptores es la de representar los conceptos principales tratados en los artículos publicados y facilitar su recuperación. La idea es que cada concepto esté representado por un único término, y que cada término represente un único concepto con la finalidad de resolver los problemas de sinonimia, polisemia y ambigüedad que comporta el lenquaje natural. El MeSH se divide en dos partes:

La primera es la lista alfabética de descriptores aceptados con las referencias cruzadas de sinónimos y términos relacionados que nos indica cuál es la palabra clave a utilizar; así, por ejemplo, si buscamos la palabra cancer, la lista nos remite a la palabra neoplasms que es el descriptor adecuado. Debajo de cada entrada podemos encontrar una pequeña nota histórica sobre el año de introducción del descriptor en el tesauro y otros descriptores utilizados para este concepto. Un ejemplo significativo con respecto a la búsqueda "bladder tumor" (tumor de vejiga) muestra la importancia de usar los términos MeSH:

Bladder cancer [All Fields (en todos los campos indexados)] --> 6656 citas Bladder Neoplasms [MeSH Terms (términos MeSH)] -->21282 citas

La segunda parte del MeSH es la clasificación jerárquica ("Tree Structures") que agrupa a todos los descriptores de la lista alfabética en 15 grandes categorías temáticas. Estas categorías se subdividen al mismo tiempo en subcategorías, en donde los descriptores se haLlan ordenados jerárquicamente del más general al más específico. Los descriptores llevan asociados unos códigos alfanuméricos que permiten también seleccionar la información mediante dichos códigos.

La mayoría de los artículos indexados en la base de datos MEDLINE Llevan asignados de 10 a 12 descriptores o términos MeSH; los que están señalados con un asterisco o un signo " + ", Los Major Concept, indican el tema principal del artículo, y los restantes hacen referencia a aspectos tratados o discutidos en el mismo. Esta opción nos permite limitar nuestra búsqueda a los artículos que traten principalmente el tema de interés y prescindir de las otras citas irrelevantes. La aplicación práctica más interesante de la clasificación jerárquica consiste en que permite recuperar citas bibliográficas muy específicas sobre un tema, al mismo tiempo que existe la posibilidad de ir ampliando el concepto introduciendo los descriptores de categorías más generales que incluyen los términos subordinados. Por ejemplo, podríamos seleccionar sólo aquellos artículos que hicieran referencia a la Meningitis viral o bien ampliar el conjunto utilizando el descriptor más general de Meningitis que incluye a todos los restantes. Será en función del tema que nos interese buscar y de los distintos aspectos que queramos combinar que utilizaremos los descriptores que nos amplíen o bien Limiten el conjunto de citas bibliográficas.

Existe además el apartado de los subencabezamientos, o "MeSH Subheadings", que consta de 88 divisiones temáticas. Estos están relacionados con los descriptores del tesauro y permite seleccionar distintos aspectos (etiología, diagnóstico, tratamiento, cirugía) de enfermedades o patologías tratadas en la literatura médica.

Por ejemplo si buscamos información sobre la prevención y control del cáncer de mama, la combinación del descriptor BREAST NEOPLASMS y el "subheading" - Prevention and control (PC) sería la forma más idónea de obtener esta información, mientras que si cruzamos estos dos términos de forma independiente, obtenemos también esta información, pero con un mayor número de referencias bibliográficas añadidas, en las cuales aparecen estos términos pero en un contexto distinto al que nos interesa. La combinación de un descriptory un subencabezamiento es a menudo la manera más precisa de acotar una búsqueda.

Otros grupos de términos que forman parte del MeSH y que pueden ayudarnos en la búsqueda de información son los "Check Tags" (Rótulos), "Publication Types" (Tipo de publicación) y los "Geographics".

Los "Check Tags" son un grupo de términos con atributos muy extensos como HUMAN, MALE, CASE REPORT, que pueden resultar muy útiles para delimitar el tema; en el supuesto de que no nos interesan los artículos experimentales, podríamos muy bien eliminarlos del resultado final utilizando el "Check Tag" HUMAN. También a la inversa, podríamos seleccionar sólo los artículos de investigación mediante el "Check Tag" ANIMAL.

Los "Publication Types" que caracterizan la forma más que el contenido de un artículo nos indican, por tanto, cuándo se trata de un editorial, un "randomized controlled trial" o estudio aleatorizado, cartas al editor, "guidelines" o guías de práctica, "review" o revisiones bibliográficas de todo lo que se ha publicado sobre un tema, etc.

Los "Geographics" incluyen términos que identifican regiones geográficas, continentes, países, estados y ciudades. Estos des criptores pueden utilizarse para restringir la búsqueda del concepto que nos interesa con una zona geográfica específica, por ejemplo as ma y Canada.

Los artículos que pasan a formar parte del Medline, en la medida que Llegan a la NLM., son sometidos a un proceso de análisis por sus expertos en bibliotecología, los cuales asignan los términos $\mathrm{MeSH}$ a dichos artículos. Mientras sucede lo antedicho, estos artículos conforman una base transitoria denominada PreMedline, esto es, artículos que se encuentran a la espera de los términos MeSH que definen su contenido. Que esté disponible el PreMedline a la consulta es uno de los puntos importantes a la hora de seleccionar el servidor de acceso al Medline en Internet. Este y otros aspectos importantes para la evaluación de dichos servidores serán abordados en la próxima entrega de este tutorial.

\section{Dr. Daniel Luna}

Servicio de Clínica Médica. Hospital Italiano de Buenos Aires. 